\title{
Predicting fetal distress and admission to neonatal intensive care unit in patients with fetal growth restriction by nomogram: A retrospective cohort study in China
}

\author{
Xiaobin Chen ${ }^{1}$, Yuan Chen ${ }^{1}$, Ying Jiang ${ }^{1}$, tian dong ${ }^{1}$, Juan Li $^{1}$, Mengmeng Yang ${ }^{1}$, baihui \\ zhao $^{1}$, and Qiong Luo ${ }^{1}$ \\ 1Zhejiang University School of Medicine Women's Hospital
}

November 9, 2020

\begin{abstract}
Objective: The purpose of this research was to establish prediction model of fetal distress risk and admission to neonatal intensive care unit(NICU) risk of patients with fetal growth restriction(FGR). Design: Case-control study, a retrospective analysis. Setting: Women's Hospital, School of Medicine, Zhejiang University in China. Population: 930 patients who were diagnosed with FGR were selected, and using fetal distress and admission to NICU as outcome.. Methods: Using lasso regression and multivariable logistic regression analysis established the nomogram prediction model of fetal distress risk and admission to NICU risk. Discrimination, calibration and clinical usability of the predicting model were respectively adopted. Internal validation was assessed using the bootstrapping validation. Main Outcome Measures: Nomograms were established for Predicting fetal distress and admission to neonatal intensive care unit in patients with FGR. Results: We found that six identified factors associated with fetal distress of patients with FGR. Four independent predictors were selected for admission to NICU of patients with FGR. The delivery method of cesarean section increased the above risks. Two nomograms were developed and verified accordingly. The two models had good discrimination and good calibration respectively. The decision curve analysis performed that the clinical usability and benefits of the nomograms were the range of $3 \%-75 \%$ and $17 \%-95 \%$. Conclusion: Two nomograms were the first to predict fetal distress and admission to NICU of patients with FGR. Establishing effective predictive models based on independent predictors could help early diagnosis and evaluation of fetal distress and admission to NICU in patients with FGR.
\end{abstract}

\section{Introduction}

Fetal growth restriction (FGR) is one of the common complications of obstetrics and the second leading cause of perinatal death. It has specific clinical features, short-term and long-term risks. ${ }^{1-3}$. Current research showed that its occurrence was affected by many factors, including maternal factors ${ }^{4}$, placental and umbilical cord factors $^{4,}, 5$, fetal factors ${ }^{6}$, genetic factors ${ }^{7-9}$, etc.

Fetal factors included multiple pregnancies, fetal chromosomal abnormalities, fetal malformations and other factors, and clinical intervention was of little significance. Owing to lack of effective treatment, the outcome of fetus was the early termination of pregnancy. However, focusing on maternal diseases, placenta and umbilical cord factors, there are currently a large number of studies to improve the prognosis of the fetus for FGR patients at risk of adverse outcome ${ }^{5}, 10,11$, but there were lack of effective predictive models to predict the occurrence of fetal distress and admission to neonatal intensive care unit (NICU) for FGR patients. Therefore, our research constructed two new predictive models. A visual and simple nomogram evaluated its risk and provided more reliable evidence for the next active treatment.

\section{Patients and methods}




\section{Patients' characteristics}

Retrospective analysis of 930 patients were delivered with FGR in the obstetrics and gynecology hospital affiliated from January 1, 2014 to December 30, 2019 at Women's Hospital, School of Medicine, Zhejiang University, in Hangzhou. We selected maternal features as follows: age, pregnancy times, birth times, body mass index (BMI) before delivery, weight gain during pregnancy, gestational week, delivery method, hypertensive disorders of pregnancy(HDP), intrahepatic cholestasis of pregnancy(ICP), gestational diabetes mellitus(GDM), hypothyroidism, Placenta previa, thrombosis, anticardiolipin syndrome, abnormal cord blood flow(absent or upside down), oligohydramnios, recurrent spontaneous abortion (RSA), using prednisone, using aspirin, using low molecular weight heparin. The following information of the offspring was obtained: sex, fetal distress, admission to NICU. We selected information about fetal distress diagnosed by obstetricians and admission to NICU from the medical records of the hospital, as the outcome of the study. The most widely adopted diagnostic criteria of fetal FGR is an estimated fetal weight (EFW) below the 10th percentile for the gestational age ${ }^{2}$. Exclusion criteria included multiple pregnancies, cases of incomplete information. Among them, there were 150 cases of fetal distress and 780 cases without fetal distress. There were 478 cases of neonates entering into the NICU and 452 cases as a control. Selecting $70 \%$ of the total cases randomly was for internal validation (Figure 1A).

\section{Statistical analysis}

Statistical analyses of all data, which were represented by counting data, performed using R software (Version 3.6.0; https://www.R-project.org). The least absolute shrinkage and selection operator (LASSO) $\operatorname{method}^{12}$ was used to screen out the clinical characteristics that it was best to predict the risk factors of fetal distress and entering into NICU, and then multivariate logistic regression analysis was used to build two predicting models of the risk factors of fetal distress and entering into NICU, $\mathrm{P}<0.05$ was considered statistically significant. Two predicting models of nomogram were formulated based on the results of logistic regression analysis and by using R software. Discrimination of the two predicting models of nomogram were assessed by the concordance index (C-index) and receiver operating characteristic (ROC) curve. Bootstrapping validation with 1,000 resample were used for calculating a relatively corrected C-index. Selecting $70 \%$ of the total sample size randomly was as internal validation. Internal validation was assessed using the bootstrapping validation. Calibration and clinical usability of the two predicting models were respectively adopted by calibration curves and decision curve analysis. Decision curve analysis is a novel method that is better than the traditional decision analytic techniques to evaluate prediction models ${ }^{13}$. Using the ROC curve and calibration to execute the Internal validation.

\section{Results}

\section{Lasso regression analysis results}

We screened out the variables using the offspring outcome of fetal distress by lasso regression analysis. Six optimal variates were selected from the twenty-one variables as follows: age, gestational week, delivery method, placenta previa, abnormal cord blood flow (absent or upside down), LMWH (Figure 1B and C). Table1 lists the patients' characteristics of the Chinese population among the six independent predictors. According to the outcome of admission to NICU, nine optimal variates were screened out among the twentyone variables using lasso regression analysis, for instance pregnancy times, gestational week, delivery method, HDP, ICP, oligohydramnios, RSA, using prednisone, newborn sex (Figure 1D and E). Table2 lists the information of nine variables.

\section{Prediction model Construction}

Selecting six optimal variates about fetal distress risk were analyzed by multivariable logistic regression analysis. The results were given in Table 3 , the prediction model that merged the above six independent predictors was presented using the nomogram (Figure 2A). Selecting nine optimal variates about the risk of admission to NICU were performed by multivariable logistic regression analysis. The results listed in Table4. The prediction model that amalgamated the above four independent predictors was revealed as the 
nomogram (Figure 2B).

\section{Predictive Accuracy of the Nomogram}

The C-index of the nomogram predicted by the risk of fetal distress was 0.733(950.718 through bootstrapping validation. Simultaneously, The C-index of the prediction nomogram of admission to NICU risk was 0.794 (95through bootstrapping validation.

Furthermore, our models demonstrated good discriminative ability in both the total (AUC of prediction model one: 0.733, Figure3A. AUC of prediction model two: 0.794, Figure 3B) and internal Validation (AUC of prediction model one: 0.727, Figure3C. AUC of prediction model two: 0.801, Figure 3D ) cases, which performed a good prediction capability in the risk of fetal distress and risk of admission to NICU nomogram.

\section{Calibration of the Nomogram}

The calibration curves of the prediction nomogram using to predict the fetal distress risk in FGR patients demonstrated a good agreement in the total (Figure 4A) and the internal Validation (Figure 4B) cases. At the same time, the calibration curves of the prediction of admission to NICU risk nomogram in FGR patients proved a good agreement in the total (Figure 4C) and the internal Validation (Figure 4D) cases.

\section{Decision Curve Analysis of the Nomogram}

The decision curve analysis (DCA) was used to perform the clinical usability and benefits of the nomogram. As shown in Figure 5A, the DCA showed that if the threshold probability of fetal distress in a patient was the range of $3 \%-75 \%$, separately, using this fetal distress risk nomogram to predict the risk of fetal distress adds more benefit than the scheme. The DCA presented that if the threshold probability of admission to NICU was the range of 19\%-97\% in Figure 5B,respectively, using this the admission to NICU risk nomogram could predict the risk of admission to NICU adds more benefit than the scheme. Net benefit in this range was compared to several overlaps on basis of the fetal distress risk nomogram and admission to NICU risk nomogram.

\section{Discussion}

With the development of medical research in recent years, nomogram is a new type of multi-factor statistical method, which has better advantages than the total statistical method. It is widely applied in the medical fields and provides a visual basis for clinical work. Our study was the first to use nomogram to explore the risk of fetal distress and admission to NICU in patients with FGR in china. Using a novel prediction tool that was the lasso regression screened out more influential and available variables from the research factors in patients with FGR.

At present, FGR is the most concerned disease that affects fetal outcome in the field of fetal medicine ${ }^{14}$. In the early stage, Barker firstly proposed a hypothesis which was "fetal origins of adult hypothesis"15, 16 . With the continuous research and development of the disease, it gradually transformed into "developmental origins of health and disease" $17,{ }^{18}$.An unhealthy maternal intrauterine environment not only affected the growth and development of the fetus, but also caused adverse consequences for the fetus ${ }^{19}$. Therefore, owing to its effective prenatal monitoring, timing and method of pregnancy termination are particularly important. However, there are still great controversies about FGR management due to the lack of effective and gold standard.

Our study started from the maternal disease to explore the outcome of the fetus, which has important clinical value. In the prediction model one, six independent variates were presented as predictors of fetal distress, and nine optimal predictors for staying into the NICU. With the increase of pregnant women's age, the decrease of gestational week, abnormal cord blood flow during pregnancy, the risk of fetal distress has increased in the fetus of FGR patients. Among them, the risk of fetal distress in FGR patients at the age of 40 years old was significantly higher than the others at the age of 35 years old (OR $=4.058,95 \%$ CI 1.872-8.622,P $<0.001)$. And abnormal cord blood flow also increased its risk obviously $(\mathrm{OR}=7.563$, 95\% CI 3.653-16.146, $P<0.001)$. FGR patients with placenta previa could reduce the occurrence of fetal 
distress $(\mathrm{OR}=0.330,95 \%$ CI $0.094-0.878, P=0.046)$. At present, there is no clear and sufficient evidence to prove that LMWH plays a role in the treatment of fetal growth restriction, so further research is still under way. Our study found that the application of LMWH during pregnancy could reduce the incidence of fetal distress $(\mathrm{OR}=0.554,95 \%$ CI $0.331-0.895, P=0.020)$, but not affecting the risk of fetal entered into the NICU by lasso regression analysis. Therefore, we recommend that LMWH could reduce the incidence of fetal distress, which is consistent with the latest research results ${ }^{3,20}$. For the use of clinical aspirin, a metaanalysis of research randomized controlled trials had shown that aspirin decreased the risk of fetal growth restriction ${ }^{21}$. We used the lasso regression to screen out variates, indicating that aspirin did not decrease the risk of fetal distress for fetal growth restriction. Our research tentatively studied that the gestational week decreases would increase the incidence of admission to NICU after birth $(P<0.001)$, which was similar to the research ${ }^{22}$. The fetus of FGR patients with HDP, ICP was easier to entry into the NICU $(P=0.032, P$ $=0.011)$. The use of prednisone during pregnancy did not reduce the incidence of admission to NICU $(P$ $=0.120$ ). For the more, it is interesting that children to be born for FGR patients whose sex is a boy was not significantly related to the incidence of staying in the $\operatorname{NICU}(P=0.244)$.

Regarding to the delivery method of FGR patients, it is not an absolute indication of cesarean section. When the cord blood flow was abnormal, it was recommended to terminate the pregnancy by cesarean section ${ }^{23}$. However, there was still a lot of controversy about the timing of delivery and the method of delivery in various countries ${ }^{23-26}$. The innovative findings of this study were that vaginal delivery, compared with cesarean delivery, could reduce the incidence of fetal distress and admission to NICU for FGR patients $(P<0.001, P$ $<0.001)$ ). A foreign study showed that most patients with FGR achieved vaginal delivery, the terrible fetal outcome did not increase ${ }^{27}$. Even one research advocated vaginal delivery ${ }^{28}$. Therefore, we recommend that FGR patients chose vaginal delivery without serious emergency complications, and we monitored the labor process during delivery.

The independent predictors of two nomograms were developed based on prediction model one and two. We could add up the single points corresponding to the independent predictors of patients with FGR to get the total points. Finally, we got the probability of risk of fetal distress or admission to NICU. It was easier and more intuitive for clinicians to understand its risks. For example, the nomogram of prediction model two, a FGR patients of 34 gestational weeks (about 83 point), using the cesarean section to terminate pregnancy (about 40 point), without HDP ( 0 point) and ICP ( 0 point). The total points are 120 points, and the corresponding risk of admission to NICU is about $78 \%$. At the same time, when we verified two models, we found that these had good discrimination and calibration power. The internal verification results are consistent with the previous ones. When verified two models, we found that these had good discrimination and calibration power. The decision curve analysis suggested two models had better clinical application value. The internal verification results are consistent with the previous ones.

The current shortcoming of this study is that the timing of LMWH treatment and the timing of drug withdrawal was not studied, so further research is needed. In summary, the establishment of an effective predictive model is the key to prenatal management of the fetal outcome of FGR patients and provides a reliable basis for clinicians. The further treatment can reduce the occurrence of adverse maternal and infant outcomes.

\section{Conclusion}

Our research showed that six identified factors associated with fetal distress and four independent predictors were selected for admission to NICU of patients with FGR. The application of LMWH during pregnancy could reduce the incidence of fetal distress. The delivery method of cesarean section increased the above risks. Two nomograms were the first to developed and verified which had good discrimination and good calibration respectively. They are valuable for clinical prediction and practicality. Establishing effective predictive models based on independent predictors could help early diagnosis and evaluation of fetal distress and admission to NICU in patients with FGR.

\section{Disclosure of interests}


The authors declare that they have no potential conflicts of interest exist, including the relevant financial, personal, political, intellectual or religious interests.

\section{Contribution to authorship}

XB Chen and Y Chen contributed to the data analysis, chart making, writing-reviewing and editing of the study. Data management and curation were by Y Jiang. Data collection was by T Dong. Chart making was by J Li. Software selection was by MM Yang. BH Zhao and Q Luo contributed to the concept, design, data collection, chart making, writing- reviewing and editing in the study and take responsibility for the accuracy of the data analysis.

\section{Ethical Approval}

This paper was approved by medical Ethics Committee of Women's Hospital, School of Medicine, Zhejiang University (Ethical batch number:IRB-20200283-R) on October 26, 2020.

\section{Funding}

This paper was supported by the National Key R\&D Program of China (2018YFC1002702), the Natural Science Foundation of Zhejiang Province of China (Grant No. LY20H040009), the Key R\&D Program of Zhejiang Province of China (No.2018C03010) and Zhejiang Medical and Health Technology Project (No.2018244300).

\section{Data sharing statement}

The data that support the findings of this study are available from the corresponding author.

\section{Acknowledgments}

We express our gratitude to the patients who participated in this study.

\section{References}

1. Frøen JF, Gardosi JO, Thurmann A, Francis A, Stray-Pedersen B. Restricted fetal growth in sudden intrauterine unexplained death. Acta obstetricia et gynecologica Scandinavica. 2004 Sep;83(9):801-7.

2. ACOG. ACOG Practice Bulletin No. 204: Fetal Growth Restriction. Obstetrics and gynecology. 2019 Feb;133(2):e97-e109.

3. Martins JG, Biggio JR, Abuhamad A. Society for Maternal-Fetal Medicine (SMFM) Consult Series \#52: Diagnosis and Management of Fetal Growth Restriction. American journal of obstetrics and gynecology. 2020 May 12.

4. Sharma D, Shastri S, Sharma P. Intrauterine Growth Restriction: Antenatal and Postnatal Aspects. Clinical medicine insights Pediatrics. 2016;10:67-83.

5. Nassr AA, Abdelmagied AM, Shazly SA. Fetal cerebro-placental ratio and adverse perinatal outcome: systematic review and meta-analysis of the association and diagnostic performance. Journal of perinatal medicine. 2016 Mar;44(2):249-56.

6. Denbow ML, Cox P, Taylor M, Hammal DM, Fisk NM. Placental angioarchitecture in monochorionic twin pregnancies: relationship to fetal growth, fetofetal transfusion syndrome, and pregnancy outcome. American journal of obstetrics and gynecology. 2000 Feb;182(2):417-26.

7. Chelbi ST, Wilson ML, Veillard AC, Ingles SA, Zhang J, Mondon F, et al. Genetic and epigenetic mechanisms collaborate to control SERPINA3 expression and its association with placental diseases. Human molecular genetics. 2012 May 1;21(9):1968-78.

8. Gascoin-Lachambre G, Buffat C, Rebourcet R, Chelbi ST, Rigourd V, Mondon F, et al. Cullins in human intra-uterine growth restriction: expressional and epigenetic alterations. Placenta. 2010 Feb;31(2):151-7. 
9. Shen K, Moroco JA. The Src family kinase Fgr is a transforming oncoprotein that functions independently of SH3-SH2 domain regulation. 2018 Oct 23;11(553).

10. Verfaille V, de Jonge A, Mokkink L, Westerneng M, van der Horst H, Jellema P, et al. Multidisciplinary consensus on screening for, diagnosis and management of fetal growth restriction in the Netherlands. BMC pregnancy and childbirth. 2017 Oct 16;17(1):353.

11. Tanaka H, Furuhashi FH, Toriyabe K, Matsumoto T, Magawa S, Nii M, et al. Management of fetal growth restriction using the contraction stress test: a case-control study. The journal of maternal-fetal \& neonatal medicine : the official journal of the European Association of Perinatal Medicine, the Federation of Asia and Oceania Perinatal Societies, the International Society of Perinatal Obstet. 2019 Oct;32(19):3221-5.

12. Kidd AC, McGettrick M, Tsim S, Halligan DL, Bylesjo M, Blyth KG. Survival prediction in mesothelioma using a scalable Lasso regression model: instructions for use and initial performance using clinical predictors. BMJ open respiratory research. 2018;5(1):e000240.

13. Vickers AJ, Cronin AM, Elkin EB, Gonen M. Extensions to decision curve analysis, a novel method for evaluating diagnostic tests, prediction models and molecular markers. BMC medical informatics and decision making. 2008 Nov 26;8:53.

14. McCowan LM, Figueras F, Anderson NH. Evidence-based national guidelines for the management of suspected fetal growth restriction: comparison, consensus, and controversy. American journal of obstetrics and gynecology. 2018 Feb;218(2s):S855-s68.

15. Barker DJ, Osmond C. Infant mortality, childhood nutrition, and ischaemic heart disease in England and Wales. Lancet (London, England). 1986 May 10;1(8489):1077-81.

16. Walker AR, Walker BF. Fetal nutrition and cardiovascular disease in adult life. Lancet (London, England). 1993 May 29;341(8857):1421.

17. Hanson MA, Gluckman PD. Developmental origins of health and disease: new insights. Basic \& clinical pharmacology \& toxicology. 2008 Feb;102(2):90-3.

18. Haas SA, Oi K. The developmental origins of health and disease in international perspective. Social science \& medicine (1982). 2018 Sep;213:123-33.

19. Kesavan K, Devaskar SU. Intrauterine Growth Restriction: Postnatal Monitoring and Outcomes. Pediatric clinics of North America. 2019 Apr;66(2):403-23.

20. Rodger MA, Carrier M, Le Gal G, Martinelli I, Perna A, Rey E, et al. Meta-analysis of lowmolecular-weight heparin to prevent recurrent placenta-mediated pregnancy complications. Blood. 2014 Feb 6;123(6):822-8.

21. Roberge S, Nicolaides K, Demers S, Hyett J, Chaillet N, Bujold E. The role of aspirin dose on the prevention of preeclampsia and fetal growth restriction: systematic review and meta-analysis. American journal of obstetrics and gynecology. 2017 Feb;216(2):110-20.e6.

22. Inácio QAS, Araujo Júnior E. Perinatal Outcomes of Fetuses with Early Growth Restriction, Late Growth Restriction, Small for Gestational Age, and Adequate for Gestational Age. 2019 Dec;41(12):688-96.

23. Vayssière C, Sentilhes L, Ego A, Bernard C, Cambourieu D, Flamant C, et al. Fetal growth restriction and intra-uterine growth restriction: guidelines for clinical practice from the French College of Gynaecologists and Obstetricians. European journal of obstetrics, gynecology, and reproductive biology. 2015 Oct;193:10-8.

24. Lees C, Marlow N, Arabin B, Bilardo CM, Brezinka C, Derks JB, et al. Perinatal morbidity and mortality in early-onset fetal growth restriction: cohort outcomes of the trial of randomized umbilical and fetal flow in Europe (TRUFFLE). Ultrasound in obstetrics \& gynecology : the official journal of the International Society of Ultrasound in Obstetrics and Gynecology. 2013 Oct;42(4):400-8. 
25. Modi N. Time to bring fetal growth assessment into the 21st century. BJOG : an international journal of obstetrics and gynaecology. 2016 Nov;123(12):1989.

26. Lausman A, Kingdom J. Intrauterine growth restriction: screening, diagnosis, and management. Journal of obstetrics and gynaecology Canada : JOGC = Journal d'obstetrique et gynecologie du Canada : JOGC. 2013 Aug;35(8):741-8.

27. Bernardes TP, Broekhuijsen K, Koopmans CM, Boers KE, van Wyk L, Tajik P, et al. Caesarean section rates and adverse neonatal outcomes after induction of labour versus expectant management in women with an unripe cervix: a secondary analysis of the HYPITAT and DIGITAT trials. BJOG : an international journal of obstetrics and gynaecology. 2016 Aug;123(9):1501-8.

28. Horowitz KM, Feldman D. Fetal growth restriction: risk factors for unplanned primary cesarean delivery. The journal of maternal-fetal \& neonatal medicine : the official journal of the European Association of Perinatal Medicine, the Federation of Asia and Oceania Perinatal Societies, the International Society of Perinatal Obstet. 2015;28(18):2131-4.

\section{Hosted file}

Figure.pdf available at https://authorea.com/users/374193/articles/491770-predicting-fetaldistress-and-admission-to-neonatal-intensive-care-unit-in-patients-with-fetal-growthrestriction-by-nomogram-a-retrospective-cohort-study-in-china 